Ann. Génét. Sél. anim., I971, 3 (3), 357-365.

\title{
ÉTUDE DE LA PRÉCOCITÉ SEXUELLE ET DE CERTAINES CARACTÉRISTIQUES DE L'ÉJACULAT CHEZ DES VERRATS APPARTENANT A 5 TYPES GÉNÉTIQUES : PREMIERS RÉSULTATS
}

\author{
P. SELLIER (*), L. DUFOUR (**) et G. ROUSSEAU \\ avec la collaboration technique de J. Carrier (**) et A. Cyr \\ Station provinciale de Recherches agricoles de La Pocatière \\ Ministère de l'A griculture du Québec (Canada)
}

\section{RÉSUMÉ}

L'expérience dont les premiers résultats sont rapportés ici concerne la mise en comparaison de 20 jeunes verrats appartenant à 5 types génétiques (Yorkshire, Landrace, Landrace $\times$ Yorkshire, Hampshire $\times$ Yorkshire, Hampshire $\times$ Landrace $)$ pour l'âge et le poids à la 1 re collecte de semence et pour cinq caractéristiques de l'éjaculat (volumes total et fluide, proportion de matériel gélatineux, concentration et nombre total de spermatozoïdes). Pour chaque verrat ces caractéristiques ont été mesurées sur 10 éjaculats successifs, obtenus avec une fréquence de collecte hebdomadaire, la période de contrôle commençant à poids vif constant $(138 \mathrm{~kg})$.

L'effet "type génétique " est hautement significatif pour l'âge et le poids à la 1 re collecte. L'analyse des composantes de la variance indique que des différences significatives existent entre verrats de même type génétique pour les caractéristiques de semence. Un petit nombre de différences significatives entre types génétiques sont mises en évidence; nos résultats suggèrent en particulier que les verrats issus de croisements présentent une production accrue de spermatozoïdes. Les corrélations entre variables, calculées intra-verrat, sont également données.

\section{INTRODUCTION}

Les facteurs de variation non génétiques de la précocité sexuelle et des caractéristiques de semence chez le Verrat ont été déjà l'objet de plusieurs analyses. Par contre, l'information sur la variabilité génétique de ces caractères reste limitée.

$\left(^{*}\right)$ Adresse permanente : Station de Génétique quantitative et appliquée, Centre national de Recherches zootechniques, I.N.R.A., 78-Jouy-en-Josas.

(**) MM. I. DUfour, agronome-professeur, G. Rousseau, agronome-professeur et J. Carrier, technologiste agricole, font partie du personnel de l'Institut de Technologie agricole de La Pocatière, Ministère de l'Agriculture du Québec. 
Des différences entre types génétiques (races, lignées consanguines, croisements) pour la précocité sexuelle du Verrat sont mises en évidence par GREEN et Winters (I944), Andrews et Warwick (I949), Wiggins et al. (I95I), HaUSER et al. (I952), Nrwa et al. (I959), MC FEE et EBben (I967), SMidT et Roth (I970); l'étude de la variation génétique intra-race de ce caractère est abordée par HuHN (I970 a).

Etudiant un certain nombre de caractéristiques de l'éjaculat chez de jeunes verrats Yorkshire et Lacombe, SwIERSTRA et RAHNEFELD (I967) n'observent aucune différence significative entre les deux races. Les résultats obtenus par Hauser et al. (I952) dans une expérience de croisement entre des lignées Poland China, Hampshire et Duroc suggèrent que le volume et la concentration de l'éjaculat, ainsi que la motilité des spermatozoïdes, ne sont pas affectés de façon sensible par l'effet d'hétérosis. Par ailleurs, selon $\mathrm{C}_{L_{\mathrm{E}} \mathrm{M}}$ et al. (I967), la variance génétique additive du nombre de spermatozoïdes dans l'éjaculat parâ̂t être importante en race Yorkshire mais cette estimation d'héritabilité est peu précise $\left(h^{2}=0,64 \pm 0,45\right)$; des valeurs de 1'héritabilité de certaines caractéristiques de semence sont également données par HuHN (I970 b).

L'objet de cette expérience est la mise en comparaison de jeunes verrats appartenant à 5 types génétiques et entretenus dans les conditions d'un centre d'insémination artificielle pour l'âge et le poids à la I $^{\text {re }}$ collecte de sperme et pour la production quantitative de semence.

\section{MATÉRIEL ET MÉTHODES}

$$
\text { I. - Matériel animal }
$$

Les 20 verrats utilisés, nés entre mai et juillet 1969, représentent les 5 types génétiques suivants : Yorkshire $(Y Y)$, Landrace $(L L)$, Landrace $\times$ Yorkshire $(L Y)$, Hampshire $\times$ Yorkshire $(H Y)$, Hampshire $\times$ Landrace $(H L)$. Les 3 premiers groupes comprennent chacun deux paires de pleins-frères, les groupes $H L$ et $H Y$ sont constitués de 4 animaux provenant de 3 portées différentes. Les verrats $Y Y$ et $L L$ sont nés à la Station même; les sujets croisés proviennent soit de la Station Fédérale de Recherches de Lennoxville $(2 L Y, 4 H L, 2 H Y)$, soit d'un élevage privé $(2 L Y, 2 H Y)$ et sont arrivés à la Station à un âge compris entre 6 et 10 semaines.

\section{2. - Entrâ̂nement des verrats et contrôle de la précccité sexuelle:}

Les 20 verrats, soumis à une alimentation rationnée (deux repas par jour), ont été placés vers l'âge de 150 jours en loges individuclles dans une porcherie fermée et chauffée pendant l'hiver. L'entrainement des jeunes verrats pour l'obtention d'une collecte de sperme a commencé à un âge compris entre 160 et 170 jours (au poids moyen de $85 \mathrm{~kg}$ ). Cet entrainement consistait en leur introduction dans la salle de récolte attenant à la porcherie et munie d'un mannequin de type classique, pendant une vingtaine de minutes et à raison de 3 ou 4 essais par semaine. Le plus souvent, ayant de commencer l'entrainement des jeunes verrats, une collecte était réalisée sur l'un des verrats adultes présents à la Station. Toutes les collectes de sperme ont été effectuées à l'aide de la "main gantée " et par la même personne.

L’âge et le poids des animaux à la première collecte ont été enregistrés. La durée de la période d'entrainement nécessaire pour obtenir une récolte a été variable : deux verrats ont été collectés dès le premier essai, la plupart des autres verrats ont donné leur première récolte dans 
les trois premières semaines d'entraînement et sans qu'il soit nécessaire de recourir à des artifices particuliers pour faire monter le verrat sur le mannequin. Toutefois dans le cas de trois verrats dont le comportement sexuel était peu satisfaisant, une truie en chaleur a dû être présentée pour obtenir une première récolte.

\section{3. - Contrôle des caractéristiques de l'éjaculat}

Pour chaque verrat, l'étude des caractéristiques de semence repose sur le contrôle de 10 éjaculats successifs. Il a été retenu de débuter le contrôle à poids vif constant, le poids moyen des verrats au premier éjaculat contrôlé étant de $138,5 \pm 1,1 \mathrm{~kg}$, ce qui correspond à un âge moyen de $234 \pm 3$ jours. De ce fait avant leur première récolte contrôlée, les verrats ont été collectés un certain nombre de fois, en moyenne tous les 10 jours, la dernière récolte d'entrainement précédant de 7 jours le début du contrôle. Une fréquence hebdomadaire de collecte avait été prévue pendant la période de contrôle; en fait la répartition des 200 éjaculats contrôlés suivant le nombre $n$ de jours séparant la collecte considérée de la collecte précédente est la suivante : $n=7$ jours : 156 éjaculats; $n=6$ ou 8 jours : 32 éjaculats; $n=5$ ou $n \geqslant 9: 12$ éjaculats. Ces 12 éjaculats proviennent de trois verrats $(1 L Y, 1 H Y, 1 L L)$ qui ont manifesté une faible libido et n'ont pas été régulièrement collectés au jour fixé, certaines tentatives se révélant infructueuses. Aussitôt après la fin de la récolte, le flacon contenant la semence était transporté au laboratoire de la porcherie où étaient effectuées les mesures suivantes :

- mesure du volume total de l'éjaculat.

- mesure du volume de la fraction fluide obtenue par filtration de l'éjaculat à travers 3 épaisseurs de "toile à fromage ".

- mesure de la concentration de l'éjaculat en spermatozoïdes : elle était déduite, de la densité optique moyenne de 3 échantillons de semence diluée au $1 / 50$, mesurée à l'aide d'un colorimètre précédemment étalonné (Cox et Melrose, 1953; Young et al., 1960). La motilité de la semence fraîche a également été évaluée mais le choix d'une échelle de notation trop peu graduée n'a pas permis de réaliser une estimation satisfaisante des différences de motilité entre éjaculats; ces différences étaient d'ailleurs peu marquées.

\section{4. - Variables étudiées et analyse statistique}

Pour l'âge et le poids à la $1^{\text {re }}$ collecte, la signification statistique des différences entre types génétiques a été testée par analyse de variance et la comparaison entre les moyennes prises deux à deux a été réalisée à l'aide du test de Tukey (SNedecor et Cochran, 1967). Pour l'étude des caractéristiques de l'éjaculat, 5 variables ont été considérées :

VTOT: volume total de l'éjaculat (ml)

VFLU : volume de la fraction fluide de l'éjaculat $(\mathrm{ml})$

PGEL : proportion de matériel gélatineux (p. cent du volume total)

CONC : concentration en spermatozoïdes de la fraction fluide (millions de spermatozoïdes par $\mathrm{ml}$ )

NOMB : nombre de spermatozoïdes dans l'éjaculat (milliards de spermatozoïdes).

Les données peuvent se classer selon un schéma hiérarchique dont les 3 facteurs sont : le groupe génétique, le verrat, le rang de l'éjaculat. La signification statistique des différences entre groupes génétiques et des différences entre verrats intra-groupe génétique a été étudiée par analyse des composantes de la variance. Par ailleurs les comparaisons entre les moyennes des groupes génétiques prises deux à deux ont été réalisées en supposant fixé l'effet "groupe génétique " et en estimant l'écart-type des moyennes de groupe à l'aide du carré moyen "entre verrats intra-groupe" (SNEDECOR et Cochran, 1967). Enfin les coefficients de corrélation entre les 5 caractéristiques de l'éjaculat ont été estimés à partir des composantes résiduelles des variances et covariances.

\section{RÉSULTATS}

\section{I. - Age et poids à la première collecte de sperme}

I es valeurs moyennes observées chez les 20 verrats de l'expérience sont : I $98 \pm 5$ jours et II $8 \pm 3 \mathrm{~kg}$ respectivement pour 1'âge et le poids à la première collecte. L'effet " groupe génétique " est hautement significatif $(\mathrm{P}<\mathrm{O}, \mathrm{OI})$ pour 
les deux caractères (tableau I). Les moyennes des groupes $Y Y, L L, H L, H Y$ et $L Y$ sont respectivement $I 76,5, I 86,8, I 85,5,2 \mathrm{I} 2,0$ et 229,0 pour 1'âge à la première collecte (en jours) et I04,9, II0,6, I22,3, I22,9 et I30,5 pour le poids à la première collecte (en $\mathrm{kg})$. Les différences sont significatives $(\mathrm{P}<0,05)$ pour 1'un et l'autre caractère d'une part entre le groupe $Y Y$ et le groupe $H Y$, d'autre part entre les groupes $Y Y$ et $L L$ et le groupe $L Y$.

\section{TABLEAU I}

Test de l'effet " groupe génétique " pour l'âge et le poids à la Ire collecte de sperme

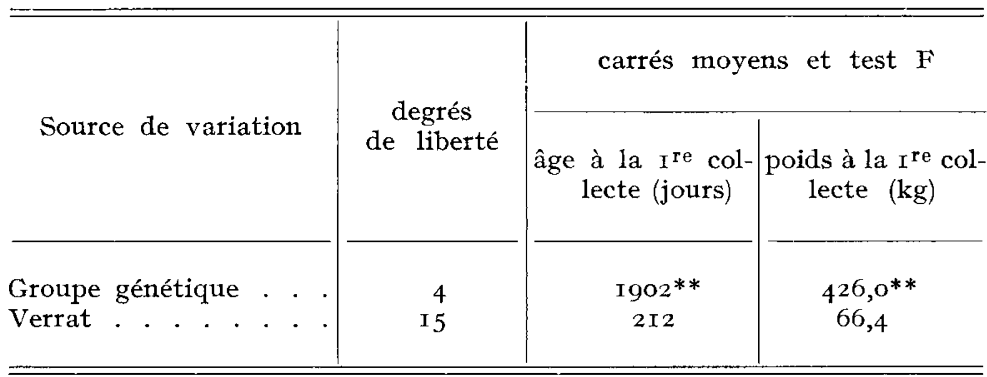

(**) effet hautement significatif $(\mathbf{P}<$ o,or $)$.

\section{2. - Caractéristiques de l'éjaculat}

Les résultats de 1'analyse des composantes de la variance figurent au tableau 2. Le carré moyen "verrat" est hautement significatif $(\mathrm{P}<0$,or $)$ pour les 5 variables, ce qui indique une forte variation entre les verrats d'un même groupe. I a composante " groupe " est significative au seuil de $5 \%$ pour la proportion de matériel gélatineux dans l'éjaculat et la concentration en spermatozoïdes de la fraction fluide de l'éjaculat. Le F relatif à la composante " groupe " est très proche du seuil de signification de $5 \%$ pour les trois autres variables, les probabilités attachées à ces valeurs de $\mathrm{F}$ étant $\mathrm{P}=0,083$ pour le volume total, $\mathrm{P}=$ 0,065 pour le volume fluide, $\mathrm{P}=0,056$ pour le nombre de spermatozoïdes dans l'éjaculat. Le test utilisé pour préciser la signification statistique des différences entre les moyennes des groupes génétiques prises deux à deux mène à des conclusions un peu différentes (tableau 3). Bien que la composante "groupe ", d'après l'analyse précédente, ne soit pas significative au seuil de $5 \%$ pour le volume fluide et le nombre de spermatozoïdes, une différence significative $(\mathrm{P}<0,05)$ est mise en évidence pour ces caractères entre le groupe Landrace et le groupe Hampshire $\times$ Landrace, à l'avantage de ce dernier; par contre, en ce qui concerne la concentration de l'éjaculat, pour laquelle la composante " groupe " est significative, aucune différence entre les moyennes comparées deux à deux n'est trouvée. De telles discordances entre tests statistiques n'ont rien d'exceptionnel dans la mesure où comme dans le cas présent, on se situe tout près des seuils de signification, dans un sens ou dans l'autre. 
TABLEAU 2

Caractéristiques de l'éjaculat : analyse des composantes de la variance

\begin{tabular}{|c|c|c|c|c|c|c|c|}
\hline \multicolumn{2}{|c|}{ Source de variation } & $\begin{array}{l}\text { degrés de } \\
\text { liberté }\end{array}$ & $\begin{array}{r}\text { V'TOT }_{(\mathrm{ml})}\end{array}$ & $\begin{array}{l}\text { VFLU } \\
\text { (ml) }\end{array}$ & $\begin{array}{c}\text { PGEL } \\
\text { (p. cent) }\end{array}$ & $\begin{array}{l}\text { CONC } \\
\text { (millions }\end{array}$ & $\begin{array}{l}\text { NOMB } \\
\text { (milliards }\end{array}$ \\
\hline Carrés moyens & $\begin{array}{l}\text { groupe }(\mathrm{G}) \\
\text { verrat }(\mathrm{V}) \\
\text { éjaculat }(\mathrm{E})\end{array}$ & $\begin{array}{l}4 \\
\text { I } 5 \\
\text { I } 80\end{array}$ & $\begin{array}{r}\text { I } 6 \text { I } 429 \\
63595 * * \\
3086\end{array}$ & $\begin{array}{rl}\text { I I } 4 & \text { I } 46 \\
4 \text { I } & 105 \\
2 & 103\end{array}$ & $\begin{array}{l}65 \text { I * } \\
\text { I } 55 * * \\
20,8\end{array}$ & $\mid \begin{array}{cc}409609 * \\
99732 * * \\
13360\end{array}$ & $\begin{array}{l}8225 \\
2799^{* *} \\
44^{6}\end{array}$ \\
\hline $\begin{array}{l}\text { composantes de } \\
\text { la variance (en } \\
\text { p. cent de la } \\
\text { variance totale) }\end{array}$ & $\begin{array}{l}\sigma_{\mathrm{G}}^{2} \\
\sigma_{\mathrm{V}}^{2} \\
\sigma_{\mathrm{F}}^{2}\end{array}$ & & $\begin{array}{l}2 \mathrm{I}, \mathrm{I} \\
52,2 \\
26,7\end{array}$ & $\begin{array}{l}23,3 \\
49,8 \\
26,9\end{array}$ & $\begin{array}{l}26,6 \\
28,8 \\
44,6\end{array}$ & $\begin{array}{l}26, I \\
29,0 \\
44,9\end{array}$ & $\begin{array}{l}x 6,6 \\
28,8 \\
54,6\end{array}$ \\
\hline
\end{tabular}

(*) effet significatif $(\mathrm{P}<\mathrm{p}, \mathrm{o5})$.

(**) effet hautement significatif $(P<$ o,or $)$.

TABLEAU 3

Caractéristiques de l'éjaculat : moyenne générale, moyennes par groupe

\begin{tabular}{|c|c|c|c|c|c|c|}
\hline \multicolumn{2}{|c|}{ variables } & $\begin{array}{l}\text { V'TOT } \\
\text { (ml) }\end{array}$ & $\begin{array}{l}\text { VFLU } \\
\text { (ml) }\end{array}$ & $\begin{array}{c}\text { PGEL } \\
\text { (p. cent) }\end{array}$ & $\begin{array}{l}\text { CONC } \\
\text { (millions } \\
\text { spz } / \mathrm{ml} \text { ) }\end{array}$ & $\begin{array}{l}\text { NOMB } \\
\text { (milliards } \\
\text { spz) }\end{array}$ \\
\hline \multicolumn{2}{|c|}{ moyenne générale } & $236 \pm 7$ & $198 \pm 6$ & ${ } 5,8 \pm 0,5$ & $501 \pm 12$ & $90,3 \pm 2,0$ \\
\hline $\begin{array}{c}\text { moyennes des } \\
\text { groupes } \\
\text { génétiques }\end{array}$ & $\begin{array}{l}\mathrm{H} \times \mathrm{Y} \\
\mathrm{Y} \times \mathrm{Y} \\
\mathrm{L} \times \mathrm{Y} \\
\mathrm{L} \times \mathrm{L} \\
\mathrm{H} \times \mathrm{L}\end{array}$ & $\begin{array}{ll}248 & (a) \\
282 & (a) \\
214 & (a) \\
139 & (a) \\
299 & (a)\end{array}$ & 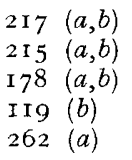 & $\begin{array}{l}\text { I3,5 }(b) \\
22,6(a) \\
\text { I6,4 }(a, b) \\
\text { 14,3 }(a, b) \\
\text { 12,5 }(b)\end{array}$ & $\begin{array}{ll}428 & (a) \\
420 & (a) \\
587 & (a) \\
634 & (a) \\
437 & (a)\end{array}$ & $\begin{aligned} 90,6 & (a, b) \\
83,2 & (a, b) \\
97,8 & (a, b) \\
70,9 & (b) \\
108,8 & (a)\end{aligned}$ \\
\hline
\end{tabular}

$(a),(b)$ : les moyennes de groupe affectées de la même lettre ne sont pas significativement différentes entre elles au seuil de $5 \%$.

Au tableau 4, figurent les coefficients de corrélation (calculés intra-verrat) entre les 5 caractéristiques de l'éjaculat. Il apparaît que le nombre de spermatozoïdes dans 1'éjaculat est plus étroitement lié à son volume qu'à sa concentration et que la proportion de matériel gélatineux dans l'éjaculat est pratiquement indépendante des autres variables, une liaison positive avec la concentration semblant toutefois exister. 
TABLEAU 4

Corrélations entre les caractéristiques de l'éjaculat (calculées intra-verrat)

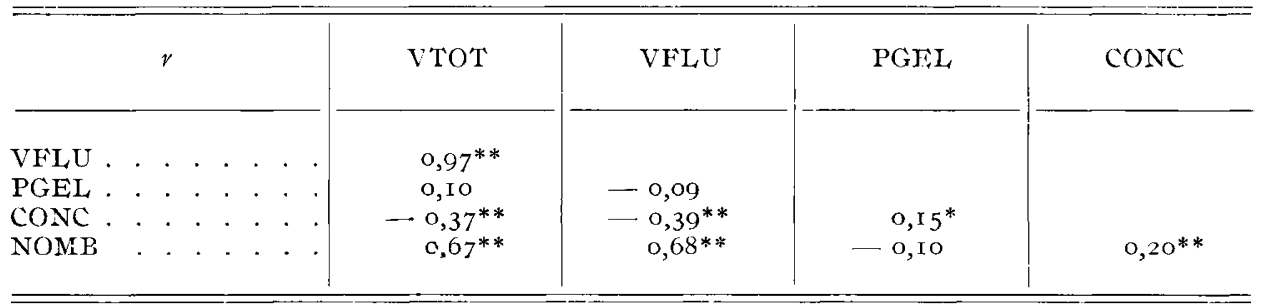

(*) $^{*}$ significatif $(\mathrm{P}<0,05)$

$(* *)$ hautement significatif $(P<0,0 I)$.

\section{DISCUSSION}

Il importe avant tout de remarquer qu'il serait imprudent d'accorder une valeur trop générale aux différences, souvent importantes, qui ont été observées entre les types génétiques étudiés. Les résultats obtenus ne sont strictement valables que pour nos échantillons et sous réserve d'erreurs d'échantillonnage dues au nombre très faible de verrats représentant chaque type génétique. On peut tout au plus dégager de ces résultats des tendances qui restent à confirmer éventuellement.

\section{I. - A ge et poids à la première collecte}

L'interprétation des données d'âge et de poids à la première collecte doit tenir compte du fait que pour obtenir cette première collecte et contrairement à ce que prévoyait le protocole initial, une truie en chaleur a du être présentée à deux verrats $H Y$ et à un verrat $L L$ dont le comfortement en salle de récolte n'était pas encourageant : ceci affecte la moyenne des groupes Landrace et Hampshir : $\times$ Yorkshire d'une erreur par défaut dont il n'est pas possible d'évaluer l'imiportance.

Par ailleurs, la collecte du premier éjaculat est seulement une manifestation de l'apparition de la maturité sexuelle, processus essentiellement graduel comme le note BANE (I959) et on ne peut prétendre donner à l'âge et au poids à la première collecte la signification de mesures de précocité de la puberté au sens strict: ces caractères présentent toutefois un intérêt non négligeable dans l'optique de l'utilisation intensive de jeunes verrats de haute valeur génétique en insémination artificielle.

Ces réserves étant faites, il y a surtout à noter qu'un effet d'hétérosis nettement défavorable s'est manifesté dans le croisement Landrace $\times$ Yorkshire pour l'âge et le poids à la première collecte et que la même tendance semble exister dans les deux autres croisements. Ce résultat est assez surprenant, d'après ce que l'on sait du phénomène d'hétérosis en général et compte tenu du fait que les données d'HAUSER et al. (I952) sur 1'âge à la I $^{\text {re }}$ collecte de verrats appartenant à des lignées consanguines ou issus de croisements simples entre ces lignées indiquent 
clairement un avantage des verrats croisés pour ce caractère, ainsi que pour le développement des organes génitaux et le stade de la spermatogénèse à l'âge de I50 jours. Cet aspect mériterait d'être abordé de nouveau sur un nombre plus grand d'animaux.

\section{2. - Caractéristiques de l'éjaculat}

Il a été retenu de débuter la période de contrôle des éjaculats à poids vif constant. Ce choix a impliqué que les verrats ont donné avant le début du contrôle entre 2 et 7 collectes selon leur précocité sexuelle. On peut penser que le fait de commencer le contrôle à des époques différentes de la vie sexuelle du verrat introduit un biais dans l'estimation de la valeur individuelle des verrats pour les caractères étudiés.

En fait SwIERstra (I969) ne trouve pas d'effet notable de " l'expérience sexuelle "sur les caractéristiques de semence de jeunes verrats âgés de 7 à Io mois. Par ailleurs, dans notre expérience, les différences entre les verrats d'un même groupe génétique sont hautement significatives pour les 5 caractéristiques de l'éjaculat et d'une façon générale, plus marquées que les différences entre les groupes génétiques étudiés. On peut noter que l'étude comparée des mêmes caractéristiques de l'éjaculat dans les races Yorshire et Lacombe ( 22 verrats par race) conduit SWIERSTRA et RAHNEFELD (I967) à une conclusion analogue, à savoir l'existence de fortes variations entre verrats de même type génétique pour les caractères de semence.

Ce fait renforce les réserves énoncées plus haut quant à la représentativité de nos échantillons d'animaux. On peut penser en particulier que les faibles volumes de semence obtenus chez les verrats Landrace sont plus une caractéristique de notre échantillon de 4 verrats que de cette race en général. Une telle tendance ne semble pas en tout cas avoir déjà été signalée dans la littérature.

La proportion de matériel gélatineux dans l'éjaculat est sensiblement plus élevée chez les verrats Yorkshire que chez les verrats des autres groupes. La valeur moyenne obtenue ici pour les verrats Yorkshire est comparable à celle trouvée par Swierstra et RAHNEFELd (I967) avec la même race et dans des conditions techniques à peu près similaires. Les proportions les plus faibles sont observées dans les groupes $H L$ et $H Y$, ce qui est à rapprocher des valeurs relativement peu élevées (I5-I6\%) observées par Dutrt et BARnharT (I959) chez des verrats de race Hampshire.

Le nombre total de spermatozoïdes émis dans l'éjaculat peut constituer une mesure quantitative de l'activité spermatogénétique du verrat, le nombre de spermatozoïdes obtenus à chaque collecte rerrésentant la plus grande partie, 85 p. cent environ, du nombre de spermatozoïdes disponibles (SWIERSTRA, I968). La valeur moyenne pour les 200 éjaculats contrôlés correspond à une émission de 90,3 milliards de spermatozoïdes par éjaculat. Selon Signorfit et Du Mesnir, DU Buisson (I970), l'augmentation de la fréquence des collectes ne modifie que très peu le nombre total de spermatozoïdes obtenus par semaine. La production hebdomadaire de spermatozoïdes observée dans le groupe Yorkshire peut donc être rapprochée de la valeur correspondante déduite des données recueillies par 
SWIERSTRA et RAHNEFELD (I967) sur des verrats de la même race et d'un âge comparable, récoltés toutes les 48 heures : les deux valeurs sont très voisines et un peu inférieures à celle observée par Signoret et du MESNiI, du Buisson (I970) sur plus de 3000 éjaculats de verrats adultes de type Large White, récoltés au rythme d'une fois par semaine. Les chiffres relatifs au nombre de spermatozoïdes dans l'éjaculat (tableau 3) mettent en évidence l'avantage des groupes croisés, notamment du groupe Hampshire $\times$ Landrace, sur les groupes Yorkshire et Landrace. L'effet d'hétérosis pour ce caractère peut être estimé à $27 \%$ dans le croisement Landrace $\times$ Yorkshire : il est lié à une manifestation d'hétérosis à la fois pour le volume fluide et pour la concentration de l'éjaculat. L'observation d'un tel effet d'hétérosis n'est pas en accord avec les conclusions d'HAUser et al. (I952), obtenues avec un autre matériel animal. Les groupes $H Y$ et $H L$ sont supérieurs aux groupes $Y Y$ et $L L$, respectivement de II et $53 \%$, pour le nombre de spermatozoïdes : l'absence d'un groupe Hampshire empêche d'évaluer dans quelle mesure ces avantages sont dus à un effet d'hétérosis ou à un effet direct supérieur de la race Hampshive pour ce caractère.

Les valeurs des corrélations entre les caractéristiques de l'éjaculat (tableau 4) diffèrent sur certains points des valeurs correspondantes, calculées intraverrat, de SwIERSTRA et RAHNEFELD (I967), notamment en ce qui concerne l'intensité des liaisons entre le nombre de spermatozoïdes dans l'éjaculat et chacune de ses deux composantes : volume fluide et concentration. Ces auteurs trouvent que le nombre de spermatozoïdes dans 1'éjaculat est étroitement lié à sa concentration (corrélation moyenne voisine de 0,8 ) et d'une façon générale assez peu lié à son volume, une forte variation entre verrats étant toutefois mise en évidence de ce point de vue (corrélations comprises entre - o,5 et 0,7 ). Une tendance pratiquement inverse est observée ici. La différence de rythme de collecte entre les deux études est peut-être à invoquer pour expliquer cette situation.

\section{CONCLUSION}

La taille très faible de nos échantillons et l'existence de fortes variations entre verrats de même type génétique limitent la portée des conclusions qui peuvent être avancées à propos des différences entre les types génétiques étudiés. Sous réserve de confirmations ultérieures, le fait le plus notable suggéré par nos résultats est 1'accroissement de la production de spermatozoïdes chez les verrats issus de croisement.

Reçu pour publication en juin 197 I.

\section{REMERCIEMENTS}

Nous tenons à remercier MM. Courot et Signoret (I.N.R.A., Nouzilly) dont les critiques et les suggestions nous ont beaucoup aidé pour la rédaction de ce texte. 


\section{SUMMARY}

\section{FIRST RESULTS OF A STUDY ON SEXUAL PRECOCITY}

\section{AND SOME SEMEN CHARACTERISTICS IN FIVE GENETIC GROUPS OF BOARS}

Twenty young boars belonging to five genetic groups : Yorkshire, Landrace, Landrace $\times$ Yorkshire, Hampshire $\times$ Yorkshire, Hampshire $\times$ Landrace were compared for age and weight at first collection of sperm and for five semen characteristics: total and strained volumes, percent gel, sperm concentration and total sperm per ejaculate. The recording of these characteristics began at a constant live weight $(138 \mathrm{~kg})$ and experimental boars were ejaculated once a week for a 10 -week period.

The effect of genetic group was significant $(\mathrm{P}<0,01)$ for age and weight at first collection Variance component analysis indicates that differences among boars within a group were significant $(P<0,01)$ in regard to semen characteristics. A few significant differences between genetic groups were found: our data suggest total sperm per ejaculate to be somewhat increased in crossbred boars. Also, correlations between semen characteristics were evaluated on a within boar basis.

\section{RÉFÉRENCES BIBLIOGRAPHIQUES}

ANDkEws F. N., WARwick İ. J., 1949. Comparative testicular development of some inbred, purebred, crossbred and linecross boars. J. Anim. Sci., 8, 603 (Abstr.).

BANE A., 1959. Some results of studies on the semen of young boars and on artificial insemination in pigs. Ann. Zootech., 8 (suppl.), I5-20.

Clem D.R., Plotka E. D., Garwood V. A., Foley C.W., I967. Heritabilities and correlations among boar semen traits. J. Anim. Sci., 26, I464-I 465 (Abstr.)

Cox C. P., Melrose D. R., I953. The calibration of a photoelectric absorptiometer for the rapid estimation of counts of spermatoza in bull semen. J. Agric. Sci., 43, 375-379.

DUtT R. H., BARNhaRT C. E., 1959. Effect of plane of nutrition upon reproductive performance of boars. J. Anim. Sci., 18, 3-13.

Green W.W., Winters I.M., I944. Histological studies of boar testes. J. Morph., 75, 29 r-30I.

Hauser E. R., Dickerson G. F., Mayer D. T., 1952. Reproductive development and performance of inbred and crossbred boars. Res. Bull. Mo. Agric. Exp. Sta., (503).

HunN U., I970 $a$. Sexualethologische untersuchungsergebnisse zur Jungeberselektion für die Schweinebesamung. Fortpfl. Haust., 6, 339-349.

Hurn U., r970 b. Hoden-und Samencharakteristika männlicher Schweine in Abhängigkeit von Judentwicklung und Vererbung. Fortpfl.Haust., 6, 350-364.

Mc Fee. A.F., Eblfin J. R., 1967. Testicular development in miniature swine. J. Anim. Sci., 26, 772776.

Nrwa T., Mizuno A., Ito S., r959. Studies on the age of sexual maturity of the boar. Ann. Zootech., 8 (suppl.), I60-I63.

Signoret J. P., du Mesnil du Buisson F., i97o. Etat actuel des techniques d'insémination artificielle porcine. Journées de la Recherche Porcine en France, I9-20 février, 29-39.

Sмmт D., Rotн E., r97o. Vergleichende Untersuchungen zur Keimdruisenentwicklung bei männlichen veredelten Landschweinen und Miniaturschweinen. Zuchthyg., 5, 65-73.

SNedecor G.W., Cochran W. G., 1967. Statistical methods ( $6^{\circ}$ édition). Iowa State University Press, Ames, Iowa.

Swierstra E. E., I968. A comparison of spermatozoa production and spermatozoa output of Yorkshire and Lacombe boars. J. Reprod. Fert., 17, 459-469.

Swierstra E. F., r969. Effects of age and sexual experience on boar semen. J. Anim. Sci., 29, 200 (Abstr.).

Swierstra E. E.,, Rahnefeld G.W., I967. Semen and testis characteristics in young Yorkshire and Lacombe boars. J. Anim. Sci., 26, I49-157.

Wiggins E.L. Warnick A.C., Grummer R.H., Casida R. H., Chapman A. B., I95I. Variation in puberty phenomena in inbred boars. J. Anim. Sci., 10, 494-504.

Young D. C., Foote R. H., Turkhemer A. R., Hafs A. D., I96o. A photoelectric method for estimating the concentration of sperm in boar semen. J. Anim. Sci., 19, 20-26. 